\title{
A proposed role for glutamine in cancer cell growth through acid resistance
}

Cell Research (2013) 23:724-727. doi:10.1038/cr.2013.15; published online 29 January 2013

\section{Dear Editor,}

Cancer cells exhibit a greatly increased level of aerobic glycolysis with accumulation of lactic acid, a phenomenon known as the Warburg effect. Apparently, survival of cancer cells requires an elaborate system for acid resistance. L-glutamine (Gln) has long been known to be essential for cancer cell growth, which is generally thought to relate to the nutritional value of Gln as carbon and nitrogen source. On the basis of our recent finding that Gln provides acid resistance for E. coli through release of ammonia, we hypothesized that the primary role of Gln in cancer cells is to fight acid, rather than provide nutrition, through enzymatic deamidation. In this letter, we provide preliminary experimental evidence that supports this hypothesis. We demonstrate that Gln helps cancer cells survive acidic stress, which is compromised by inhibition of specific glutaminase activity. Our data suggests that glutaminase inhibitors, currently under clinical trials as an anti-cancer drug, may work by countering the ability of cancer cells to survive under acidic environment. We further speculate that the general requirement of Gln in cell culture is also due to its crucial role in acid resistance.

A hallmark of cancer cell is described as the Warburg Effect, with increased aerobic glycolysis and reduced oxidative phosphorylation [1]. Even in the presence of oxygen, cancer cells convert glucose mainly into lactic acid, which, with a pK of 3.7, contributes to an acidic environment in tumor [2]. The extracellular $\mathrm{pH}$ value of human cancer tissues can be as low as 5.6 [3]. As numerous biological reactions are strictly $\mathrm{pH}$ dependent, lowered $\mathrm{pH}$ value may be detrimental to cancer cell growth. Consequently, cancer cells must be able to efficiently neutralize lactic acid to ensure normal growth. Supporting this analysis, the intracellular $\mathrm{pH}$ in tumors was reported to remain largely unchanged even when the extracellular $\mathrm{pH}$ value precipitously dropped as a consequence of cell growth [4]. This observation suggests presence of a robust acid resistance system within cancer cells. Much to our surprise, there is little description or research into the acid resistance system in cancer cells.

L-glutamine (Gln) is the most abundant free amino acid in human body [5], with its concentration higher than that of all other 19 amino acids combined. Gln is also the most abundant free amino acid in a large variety of food sources, including meat and vegetables [6]. Remarkably, cancer cells heavily depend on Gln for growth and proliferation [5], and glutamine deprivation in cell culture leads to rapid loss of cell viability [7]. Strikingly, most tumor cells consume Gln at a much higher rate compared to normal cells [8]. The prevailing view is that Gln provides carbon and nitrogen source for cell growth, as Gln is converted into L-glutamate (Glu) and ammonia through glutaminolysis [9]. Unfortunately, this common belief is contradicted by the experimental observation that, despite efficient transport systems for Glu and ammonium, Glu at any concentration, even if supplemented by $\mathrm{NH}_{4}^{+}$and ATP, failed to permit the growth of L-fibroblast [7]. Thus, we believe that the question of why Gln is required for cancer cell growth and more generally cell culture remains largely enigmatic.

In the accompanying paper [6], we report a novel bacterial acid resistance system, which relies on the release of ammonia from Gln via the enzymatic activity of glutaminases. The released ammonia neutralizes the acid in $E$. coli. This finding, together with the use of ammonia by Helicobactor polari to fight acid in the stomach [10], motivated us to hypothesize an important role for Gln in cancer cell growth. In this hypothesis, Gln is thought to facilitate cancer cell growth not just by providing carbon and nitrogen source, but more importantly, by fighting acidic stress through enzymatic release of ammonia. Our proposed hypothesis is consistent with a body of existing literatures and knowledge. Two different glutaminases (GLS) have been identified in mammalian cells: GLS1 (with two splice forms KGA and GAC [11, 12]), which is mainly expressed in the kidney, and GLS2 (also called LGA [13]), which exists mainly in the liver. The mRNA levels of GAC have been found to be elevated in various tumors [12]. In fact, GAC was shown to be essential for the growth of non-small cell lung cancer [14]. Gluta- 
$\mathbf{A}$

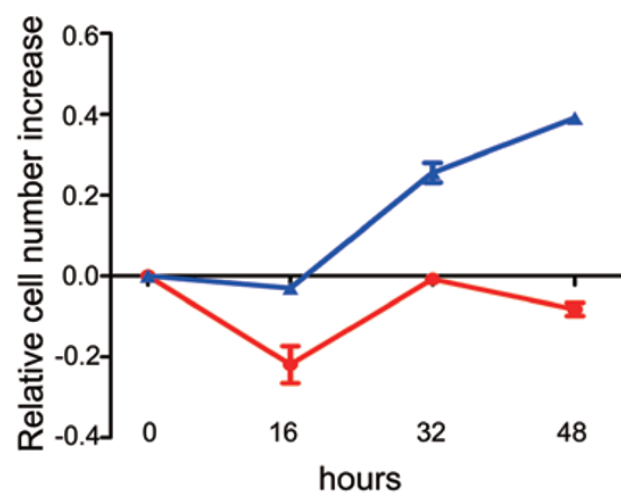

B

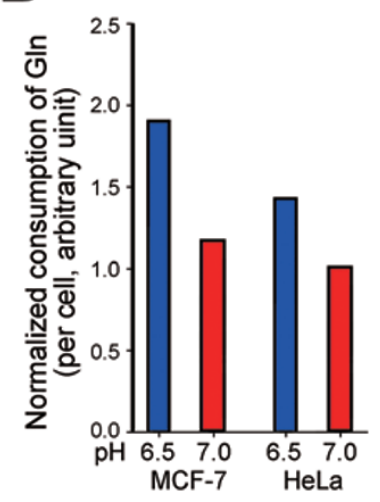

HeLa

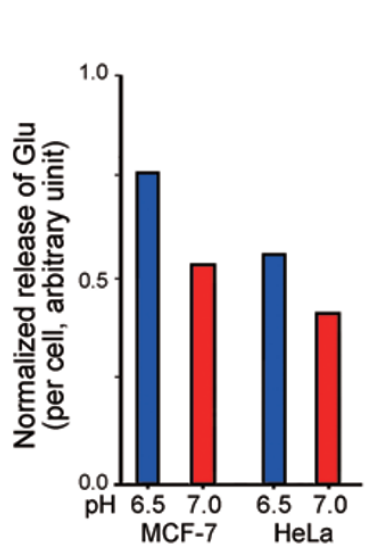

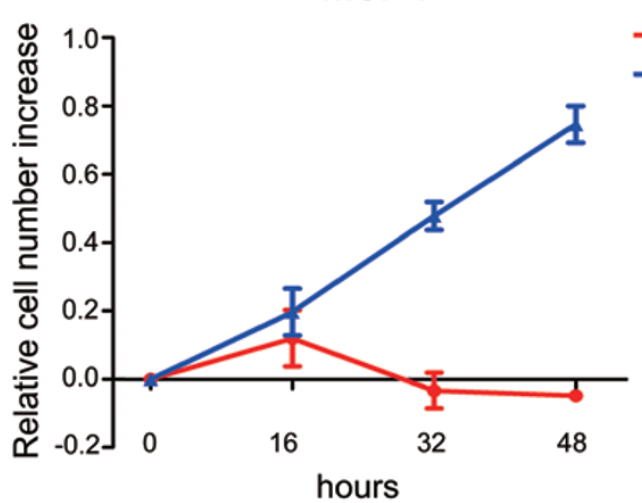

C
HeLa

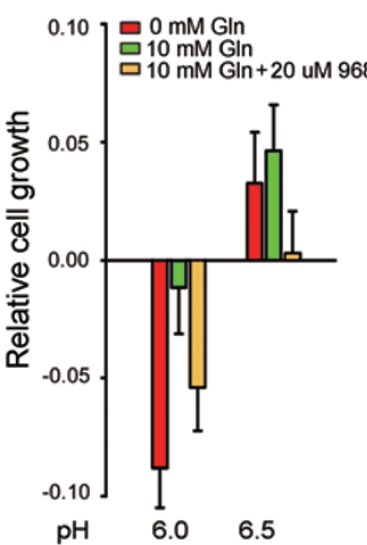

MCF-7

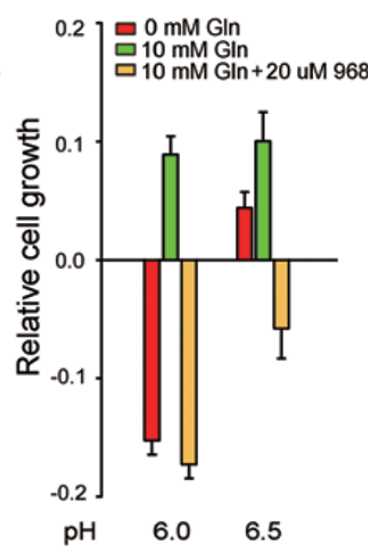

D

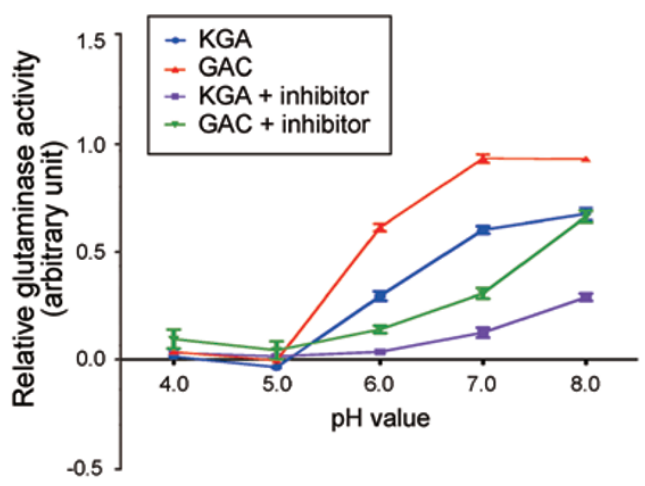

E

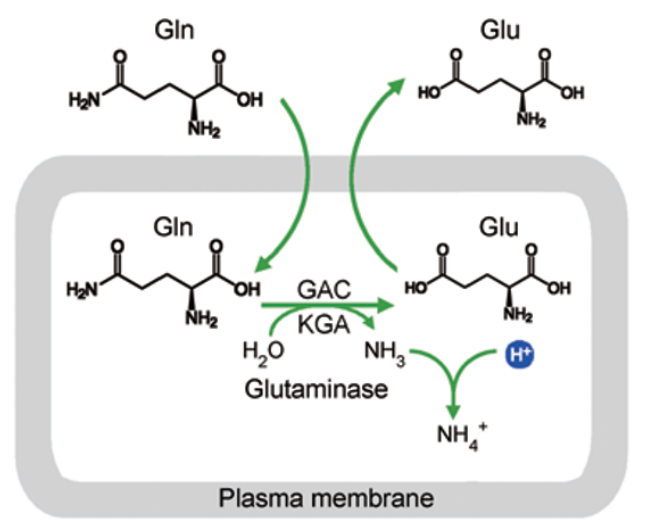

Figure 1 L-glutamine (Gln) may play an essential role in cancer cell growth through enzymatic release of ammonia for acid resistance. (A) HeLa and MCF-7 cells grow in the absence of GIn with continued supply of buffered medium. HeLa and MCF-7 cells were cultured without $\mathrm{Gln}$ at $\mathrm{pH} 6.3$ and 7.3. Culture medium was changed every $8 \mathrm{~h}$ to maintain relatively constant $\mathrm{pH}$ value. Cell density was determined by the CV staining method. (B) HeLa and MCF-7 cells consume more GIn and release more glutamate (Glu) in culture medium of lower pH. Two pH values, 6.5 and 7.0, were used. Gln and Glu concentrations were determined by mass spectrometric analysis. (C) Inhibition of glutaminase activity results in reduction of cell survival and growth under acidic environment. Relative cell growth indicates the normalized change of cell number after cell growth in different medium for $8 \mathrm{~h}$. A relative cell growth value of 0.05 means $5 \%$ additional cell growth compared to that at hour 0 . The results shown were the average of 10 independent experiments. Medium pH is buffered by 25 mM PIPES. (D) The GLS1 splice variant GAC exhibits a higher activity than KGA, especially at $\mathrm{pH}$ 6.0. Shown here is in vitro activity characterization of GAC and KGA at different pH values. The glutaminase activity was measured by the Glu dehydrogenase-based, two-step glutaminase protocol. The proteins used here contain an N-terminal truncation (residues 1-122) for better expression in $E$. coli. (E) A schematic diagram to illustrate our hypothesis on the essential role of Gln in cancer cell survival. 
minase inhibitors have been under clinical trials in the United States as a potential anti-cancer therapy [15]. It should be noted, however, these previous studies did not link the important role of glutaminase to the requirement of Gln in acid resistance for cancer cell growth.

To help validate this hypothesis, we studied the impact of Gln in cell growth using HeLa and MCF-7 cells, derived from cervical and breast cancers, respectively. In this letter, we report that, as cells grow, the extracellular environment becomes increasingly acidic, and continued cell growth requires Gln. In culture medium of lower $\mathrm{pH}$, cancer cells consumed more Gln, with more Glu accumulated in the extracellular environment. The pro-survival role of Gln under acidic $\mathrm{pH}$ is sabotaged by specific glutaminase inhibitor. These data collectively support the hypothesis of an essential role for Gln in cancer cell growth under acidic stress. Our preliminary experimental evidence is briefly outlined below.

First, we show that cancer cell lines appear to depend on Gln to maintain normal growth. HeLa and MCF-7 cells were cultured following manufacturer's protocol but with provision of varying concentrations of $\operatorname{Gln}(0,5,10$, and $25 \mathrm{mM}$ ); cell density was measured at $0,8,16,24$, 48,72 , and $96 \mathrm{~h}$. There is a strong correlation between cell growth and the presence of Gln (Supplementary information, Figure S1). In the presence of $5 \mathrm{mM}$ or higher concentrations of Gln, the growth curves appear qualitatively the same for HeLa or MCF-7 cells. By contrast, the HeLa or MCF7 cell density was considerably lower in the absence of Gln (Supplementary information, Figure S1). Reduced levels of Gln also gave rise to reduced rate of growth for HeLa and MCF-7 cells (Supplementary information, Figure S2).

Next, we measured the $\mathrm{pH}$ values of the culture medium at different time points. Consistent with the notion that cell growth results in acidification of the culture medium, the $\mathrm{pH}$ decreased over time for both cell lines (Supplementary information, Figure S3). Reflecting a reciprocal relationship to their growth curves, the culture medium for HeLa or MCF-7 cells exhibited a similar temporal decrease of $\mathrm{pH}$ values in the presence of $5 \mathrm{mM}$ or higher Gln. By contrast, the $\mathrm{pH}$ value of the HeLa or MCF-7 culture medium showed much less decrease in the absence of Gln, presumably due to lack of cell growth. In the absence of cells, the culture medium $\mathrm{pH}$ remained largely unchanged over time (Supplementary information, Figure S3).

If acidic $\mathrm{pH}$ caused by cell growth is detrimental to continued cell growth, then continued supply of buffered medium might alleviate this problem. To examine this scenario, we cultured $\mathrm{HeLa}$ or MCF-7 cells at relatively constant $\mathrm{pH}$, with medium change every $8 \mathrm{~h}$. At $\mathrm{pH} 7.3$,
HeLa and MCF-7 cells exhibited robust growth even in the absence of Gln (Figure 1A). By contrast, cell growth was considerably less at $\mathrm{pH} 6.3$ in the absence of Gln (Figure 1A). Consistent with this analysis, the presence of $10 \mathrm{mM}$ Gln allowed robust cell growth at both $\mathrm{pH} 6.3$ and pH 7.3 (Supplementary information, Figure S4). Together, our data suggest that, for HeLa or MCF-7 cancer cells, Gln might provide an efficient means of countering the acidic stress.

If Gln is used for acid resistance, then we would expect to see consumption of Gln from, and release of Glu into, the culture medium. In addition, there might be more consumption of Gln at lower $\mathrm{pH}$. To investigate this scenario, we cultured HeLa or MCF-7 cells in the presence of $0.5 \mathrm{mM} \mathrm{L}-\left[\mathrm{U}_{-}{ }^{13} \mathrm{C}_{5},-\mathrm{U}_{-}{ }^{15} \mathrm{~N}_{2}\right] \mathrm{Gln}$ and $10 \mathrm{mM}$ unlabelled Gln at $\mathrm{pH} 6.5$ or 7.0. After $8 \mathrm{~h}$, the remaining Gln in the culture medium was quantified using mass spectrometry. For both HeLa and MCF-7 cells, there is more cell growth at $\mathrm{pH} 7.0$ than at $\mathrm{pH} 6.5$ (Supplementary information, Figure S5); yet consumption of Gln at $\mathrm{pH}$ 6.5, normalized for cell density, is considerably more than that at $\mathrm{pH} 7.0$ (Figure 1B, left panel). Specifically, compared to $\mathrm{pH} 7.0, \mathrm{HeLa}$ and MCF-7 cells at $\mathrm{pH} 6.5$ exhibit $61 \%$ and $37 \%$ more consumption of Gln, respectively. Consistent with intracellular conversion of Gln to Glu, the concentrations of Glu in the culture medium increased by $39 \%$ and $33 \%$, respectively, for $\mathrm{HeLa}$ and MCF-7 cells (Figure 1B, right panel).

If conversion of Gln to Glu is required for acid resistance, then inhibition of the glutaminase activity is expected to preferentially inhibit cell growth at lower $\mathrm{pH}$. To examine this scenario, we cultured HeLa and MCF-7 cells in medium with or without glutaminase inhibitor at $\mathrm{pH} 6.0$ and 6.5. At both $\mathrm{pH}$ values, despite the presence of $10 \mathrm{mM} \mathrm{Gln}$, there were much less cells in the presence of the glutaminase GLS1-specific inhibitor 968 compared to its absence (Figure 1C). Serving as a control in these experiments, cell growth in the absence of Gln was considerably worse than that in the presence of Gln. These results also suggest that GLS1 is likely a crucial enzyme for glutaminolysis in $\mathrm{HeLa}$ and MCF-7 cells under acidic stress.

Many mammalian enzymes are active only within a narrow $\mathrm{pH}$ window. To examine the $\mathrm{pH}$ dependence of glutaminase activity, we expressed and purified the two GLS1 isoforms (KGA and GAC) to homogeneity and measured their enzymatic activity in vitro. The result clearly shows that, even at $\mathrm{pH}$ 6.0, both GAC and KGA retained significant glutaminase activity (Figure 1D). GAC exhibited a higher level of glutaminase activity than $\mathrm{KGA}$ at $\mathrm{pH}$ 6.0, 7.0, and 8.0. In particular, GAC exhibits approximately $108 \%$ higher activity compared to 
KGA at $\mathrm{pH}$ 6.0. This result suggests that the GLS1 splice variant GAC may be more important for cancer cell growth under acidic $\mathrm{pH}$.

These experimental observations serve as preliminary evidence to support our hypothesis that Gln may play an important role in cancer cell survival and growth through enzymatic release of ammonia for acid resistance (Figure $1 \mathrm{E})$. In other words, the reliance of cancer cells on Gln is not just for nutrition but more importantly also for the purpose of fighting acid stress as a result of Warburg Effect. As the first step for metabolic utilization of Gln involves the glutaminase activity, it is inherently difficult to separate the two potential functions of Gln - carbon/ nitrogen source versus acid resistance. Any molecular biology manipulation of glutaminases simultaneously affects both metabolic utilization of Gln and its role in acid resistance. Nonetheless, our finding that Gln is preferentially converted to Glu in the culture medium of lower $\mathrm{pH}$ strongly supports the acid resistance hypothesis. To the best of our knowledge, this may be the first time that an important role for Gln in cancer cell growth through acid resistance is proposed. Such a conceptual advance has important ramifications for understanding cancer cell homeostasis and for designing potential therapeutic treatment. Detailed methods are described in the Supplementary information, Data S1.

\section{Acknowledgments}

This work was supported by the Ministry of Science and Technology of China (2009CB918801), the National Natural Science Foundation of China (31130002, 31021002, and 30888001), and Beijing Municipal Commissions of Education and Science and Technology. We apologize to those colleagues whose papers are not cited due to strict reference limit.

Weijiao Huang ${ }^{1,2,3, *}$, Wooyoung Choi $^{1,2,3, *}$,
Yuling Chen, ${ }^{1,2}$ Qi Zhang ${ }^{2}$, Haiteng Deng ${ }^{1,2}$, Wei $\mathrm{He}^{2}$, Yigong $\mathrm{Shi}^{1,2,3}$

${ }^{1}$ Ministry of Education Key Laboratory of Protein Science, ${ }^{2}$ TsinghuaPeking Joint Center for Life Sciences, ${ }^{3}$ Center for Structural Biology, School of Life Sciences and School of Medicine, Tsinghua University, Beijing 100084, China

*These two authors contributed equally to this work.

Correspondence: Yigong Shi

E-mail: shi-lab@tsinghua.edu.cn

\section{References}

1 Warburg O. Science 1956; 123:309-314.

2 Tannock IF, Rotin D. Cancer Res 1989; 49:4373-4384.

3 Griffiths JR. Br J Cancer 1991; 64:425-427.

4 Gerweck LE, Vijayappa S, Kozin S. Mol Cancer Ther 2006; 5:12751279.

5 Medina MA. J Nutr 2001; 131(9 Suppl):2539S-2542S. Discussion 2550S-2531S.

6 Lu P, Ma D, Chen Y, et al. Cell Res 2013; 23:635-644.

7 Eagle H. Science 1955; 122:501-514.

8 Medina MA, Sanchez-Jimenez F, Marquez J, et al. Mol Cell Biochem 1992; 113:1-15.

9 DeBerardinis RJ, Cheng T. Oncogene 2010; 29:313-324.

10 Montecucco C, Rappuoli R. Nat Rev Mol Cell Biol 2001; 2:457-466.

11 Curthoys NP, Kuhlenschmidt T, Godfrey SS, et al. Arch Biochem Biophys 1976; 172:162-167.

12 Elgadi KM, Meguid RA, Qian M, et al. Physiol Genomics 1999; 1:5162.

13 Aledo JC, Gomez-Fabre PM, Olalla L, et al. Mamm Genome 2000; 11:1107-1110.

14 van den Heuvel AP, Jing J, Wooster RF, et al. Cancer Biol Ther 2012; 13:1185-1194.

15 Rajagopalan KN, DeBerardinis RJ. J Nucl Med 2011; 52:1005-1008.

(Supplementary information is linked to the online version of the paper on the Cell Research website.)

(a) This work is licensed under the Creative Commons Attribution-NonCommercial-No Derivative Works 3.0 Unported License. To view a copy of this license, visit http:// creativecommons.org/licenses/by-nc-nd/3.0 